\section{Pyogenic granuloma arising within capillary malformations in children: A case report and literature review}

\author{
Alessandra Rancan, ${ }^{1,2}$ \\ Alessandro Boscarelli, \\ Daniela Codrich, ${ }^{2}$ Irene Berti, ${ }^{3}$ \\ Edoardo Guida, ${ }^{2}$ Jürgen Schleef ${ }^{2}$ \\ ${ }^{1}$ Pediatric Surgery Unit, Department of \\ Women's and Children's Health, \\ University of Padua; ${ }^{2}$ Department of \\ Pediatric Surgery and Urology, Institute \\ for Maternal and Child Health - IRCCS \\ "Burlo Garofolo", Trieste; ${ }^{3}$ Department \\ of Pediatrics, Unit of Pediatric \\ Dermatology, Institute for Maternal and \\ Child Health - IRCCS "Burlo \\ Garofolo", Trieste, Italy
}

\begin{abstract}
Pyogenic granuloma (PG) is an acquired benign vascular hyperplasia. Even though PG is not so rare, its etiology is still unclear. Assuredly, an association between vascular anomalies and PG is an extremely rare finding, and a proper management of these cases is not well standardized to date. Herein, we report our experience with a giant $\mathrm{PG}$ arising spontaneously within a port-wine stain of the thorax. Previous cases reported in the pediatric literature and strategies of treatment are also discussed.
\end{abstract}

\section{Introduction}

Pyogenic granuloma (PG), also known as lobular capillary hemangioma, is an acquired benign vascular hyperplasia. The etiology of PG is still unclear, and it mainly develops in children and young adults. PG usually presents as a red papular lesion that grows rapidly, bleeds with minor trauma and tends to ulcerate. PG generally occurs on skin and mucous membranes (face, trunk, oral cavity), but it can be occasionally found in other sites such as the gastrointestinal tract or the larynx. PG can arise spontaneously, in sites of injury, after laser treatment or within capillary malformations. It can also be associated with pregnancy, as well as certain medications..$^{1-4}$ Despite its relatively high occurrence, the diagnosis and treatment of PG is not still well standardized, especially in case of uncommon presentations. Herein, we report a case of a giant PG arising spontaneously within a capillary malformation of the chest in a female child. Previous cases reported in the pediatric literature and strategies of treatment are also discussed.

\section{Case Report}

A 4-year-old girl with a history of a capillary malformation (port-wine stain type) located on the left antero-superior part of the thorax presented to our Pediatric Dermatologic Unit because of a new lesion growing on her birthmark. There was no history of trauma, laser treatment, or medications use. The lesion was painless and no signs of ulceration were present. Six months after occurrence, the patient returned referring about an extremely rapid growth of the lesion with several episodes of secretion of blood and serum. On physical examination the lesion presented as an exophytic, purple-blue, moist tumor, with an ulcerated surface (Figure 1). The ultrasonographic findings demonstrated a lobulated lesion of nearly $10 \times 16$ millimeters, with proper arterial and venous flow at the color doppler study. Patient was scheduled for surgery, and surgical excision of the lesion was performed by ligating the vascular pedicle at its base. The histologic analysis described a giant ulcerated pyogenic granuloma $(18 \times 15 \times 14$ millimeters $)$ with an internal cavernous component arising on a capillary malformation of the skin (Figure 2). Because of its extension, primary excision of the entirety of the port-wine stain was not carried out and part of the birthmark was left untreated. Unfortunately, two weeks after surgery the patient experienced a dehiscence of the surgical wound. Thus, it was treated conservatively using a spray containing silver (Hyalosilver ${ }^{\circledR}$ ) and hyaluronic acid-based dressing (Connectivine $\left.{ }^{\circledR}\right)$ with prompt resolution and good aesthetic outcome. The patient is regularly followed up for recurrence, and an eventual second-look surgery for scar revision and radical excision of the capillary malformation.

\section{Discussion}

Port-wine stains (PWS) are a type of congenital capillary malformation that presents as a homogenous deep red or purple macule at birth. They are usually unilateral and tend to persist into adulthood, causing mainly cosmetic concern. In case of eyelids and/or frontotemporal involvement, glaucoma and Sturge-Weber Syndrome always need to be ruled out because PWS are known to be associated with deeper tissue
Correspondence: Alessandro Boscarelli, Department of Pediatric Surgery and Urology, Institute for Maternal and Child Health IRCCS "Burlo Garofolo", via dell'Istria, 65/1, 34137 Trieste (TS), Italy.

Tel.: +39.040.3785372.

E-mail: tboscar@hotmail.it

Pyogenic granuloma; port-wine stain; children; case report; literature review.

Acknowledgments: The Authors would like to thank Prof. Dr. Rosanna Bussani from Institute of Pathological Anatomy, Riuniti Hospital, University of Trieste, Trieste, Italy for her support with histopathological analysis.

Contributions: Dr. Rancan, Dr. Boscarelli, Dr. Codrich, and Dr. Guida had a major role in the study design, interpretation of data, and drafting of the manuscript. Dr. Berti and Dr. Schleef supervised the study, read and approved the final manuscript.

Conflict of interest: The authors declare no potential conflict of interest.

Funding: None.

Informed consent: Consent to publish this report not obtained. The report does not contain any personal information that could lead to identification of the patient.

Please cite this article as: Rancan A, Boscarelli A, Codrich D, et al. Pyogenic granuloma arising within capillary malformations in children: A case report and literature review. Dermatol Rep 2021;13:9115.

Received for publication: 2 March 2021.

Revision received: 29 March 2021.

Accepted for publication: 8 April 2021

This work is licensed under a Creative Commons Attribution-NonCommercial 4.0 International License (CC BY-NC 4.0).

${ }^{\circ}$ Copyright: the Author(s), 2021

Licensee PAGEPress, Italy

Dermatology Reports 2021; 13:9115

doi:10.4081/dr.2021.9115

hyperplasia and/or malformations. Microscopically, PWS are composed of dilatated venules and capillaries in the dermis. An association between PWS and PG has already been reported, although it represents a sporadic finding. In fact, erythrocyte stasis may cause changes in the PWS and consequently arteriovenous malformations or tumors can develop. ${ }^{6-8} \mathrm{PG}$ frequently occurs after laser treatment, local trauma, or during pregnancy and it could present in a variety of locations. Assuredly, highly vascularized areas such as the fingers, hands, lips, tongue and face are preferred sites. So, 
it has been suggested that the spontaneous development of PG in PWS might be associated with the formation of microscopic arteriovenous anastomoses in the context of the ectatic capillaries. In clinical practice, presentation without predisposing factors is rare and it can put the physicians in alarm, especially in case of skin location due to rapid growth and macroscopic similarity with malignant lesions such as amelanotic melanoma. ${ }^{8,9}$

A literature review was conducted on PubMed, Scopus and Web of Science. Every article was analyzed in order to detect pediatric $(<18$ years old) cases. The research included the following $\mathrm{MeSH}$ terms: "pyogenic granuloma", "port-wine stain" and "nevus flammeus". A total of 97 results were found (Figure 3). One paper reported a pediatric case but detailed informations were lacking, and thus it was excluded. ${ }^{10}$ The total number of previously reported cases was 7 (Table 1). In contrast with the previously reported adult cases, predisposing factors such as manipulation or syndromic context were present in only two children. Particularly, one occurring after flashlamp-pumped pulsed dye laser treatment, ${ }^{11}$ and one in the context of phacomatosis pigmentovascularis and SturgeWeber syndrome. ${ }^{12}$ This may be consequent to a missed information (e.g., history of trauma not reported) or it may suggest a different pathogenesis in children. Interestingly, a cavernous component or the presence of microscopic arteriovenous anastomoses can be present in the capillary malformations in pediatric patients, but it could remain unknown because the diagnosis of PWS is commonly clinic and rarely demands further investigations. ${ }^{7}$ However, pathogenic mechanisms of $\mathrm{PG}$ arising on PWS are still poorly understood and we found no agreement in management and treatment strategies among authors. Undoubtedly, surgical excision of the PG with sparing of the underlying PSW appears to be the preferred treatment. Nevertheless, in 2 cases recurrence was observed with one or multiple satellite lesions. ${ }^{3,13}$ This suggests that having developed a PG on a PWS may predispose to further tumor formation, although there is no scientific evidence up to date. Since recurrence is a potential and established complication, we suggest that radical excision of the entire PWS together with the PG should be always taken into consideration when feasible. Unfortunately, PWS often involves the face or extended areas of the body, thus surgical management can be challenging. Consequently, scarifica-

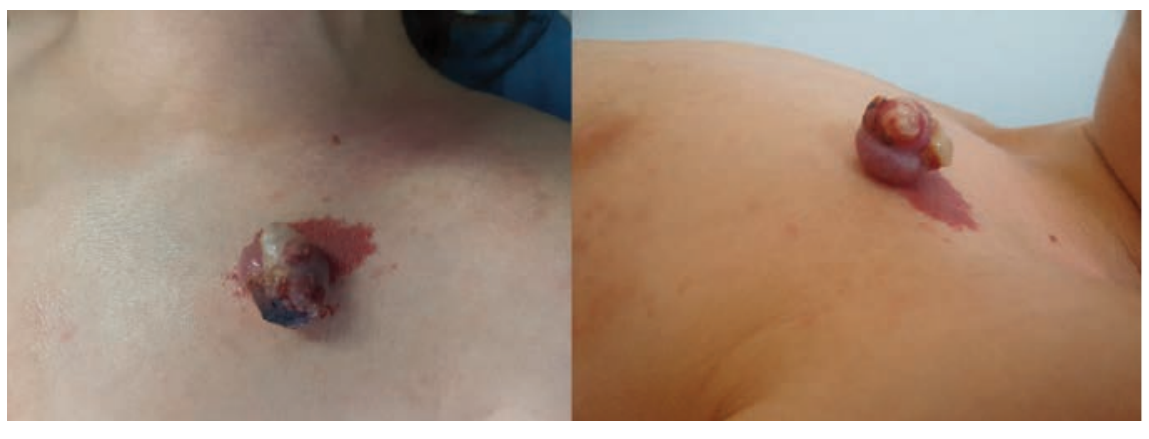

Figure 1. Close-up view of anterior (left) and lateral (right) appearance of a giant pyogenic granuloma arising within a capillary malformation of the anterior thoracic wall.

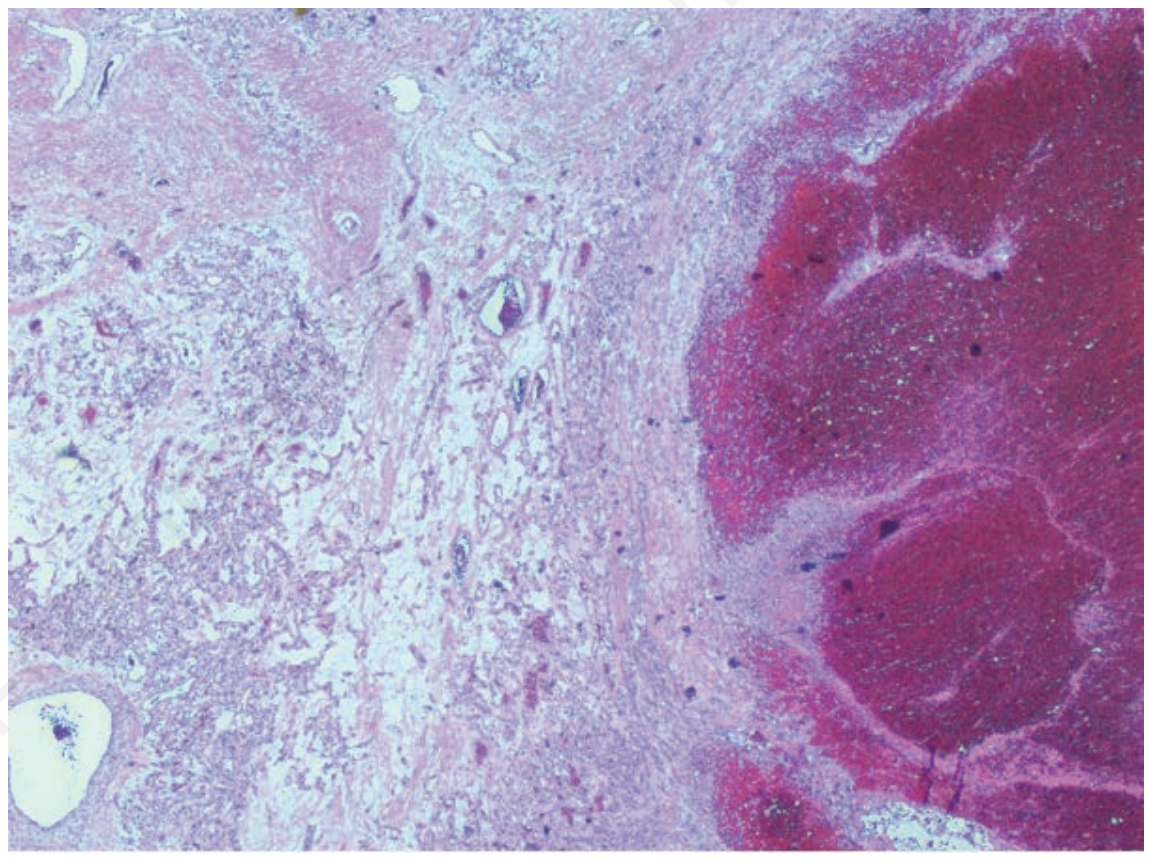

Figure 2. Microscopic image of the giant pyogenic granuloma taken through a $2.5 \mathrm{X}$ objective on hematoxylin and eosin-stained histological section.

Table 1. Previous reported cases of pyogenic granuloma (PG) arising within a port-wine-stain (PWS) in children.

\begin{tabular}{|c|c|c|c|c|c|}
\hline Reference & Age & Sex & Location(s) & Treatment & Notes \\
\hline Warner, 1968 [13] & 8-year-old & M & Sternum & Cauterization & Recurrence with multiple satellites \\
\hline Swerlick, 1983 [3] & 8-year-old & M & Shoulder/back/neck & Surgical excision of the PG & Several PGs recurring on an extended PWS \\
\hline Abd-el-Raheem, 1994 [11] & 4-month-old & M & Forehead & Surgical excision & Previous flashlamp-pumped pulsed dye laser treatment \\
\hline Hagiwara et al., 1998 [12] & 10-year-old & $\mathrm{F}$ & Forehead & Not reported & $\begin{array}{l}\text { Phacomatosis pigmentovascularis type Ilb associated } \\
\text { with Sturge-Weber syndrome }\end{array}$ \\
\hline Lee, $2000[9]$ & 16-year-old & M & Right eyebrow & Surgical excision of the PG & None \\
\hline \multirow[t]{2}{*}{ Liu, $2010[14]$} & 11-year-old & 1 & Not reported & Not reported & None \\
\hline & 14-year-old & l & Left forehead & Not reported & None \\
\hline Yang, 2013 [4] & 10-year-old & $\mathrm{F}$ & Right shoulder & Nd/YAG holmium laser & None \\
\hline
\end{tabular}




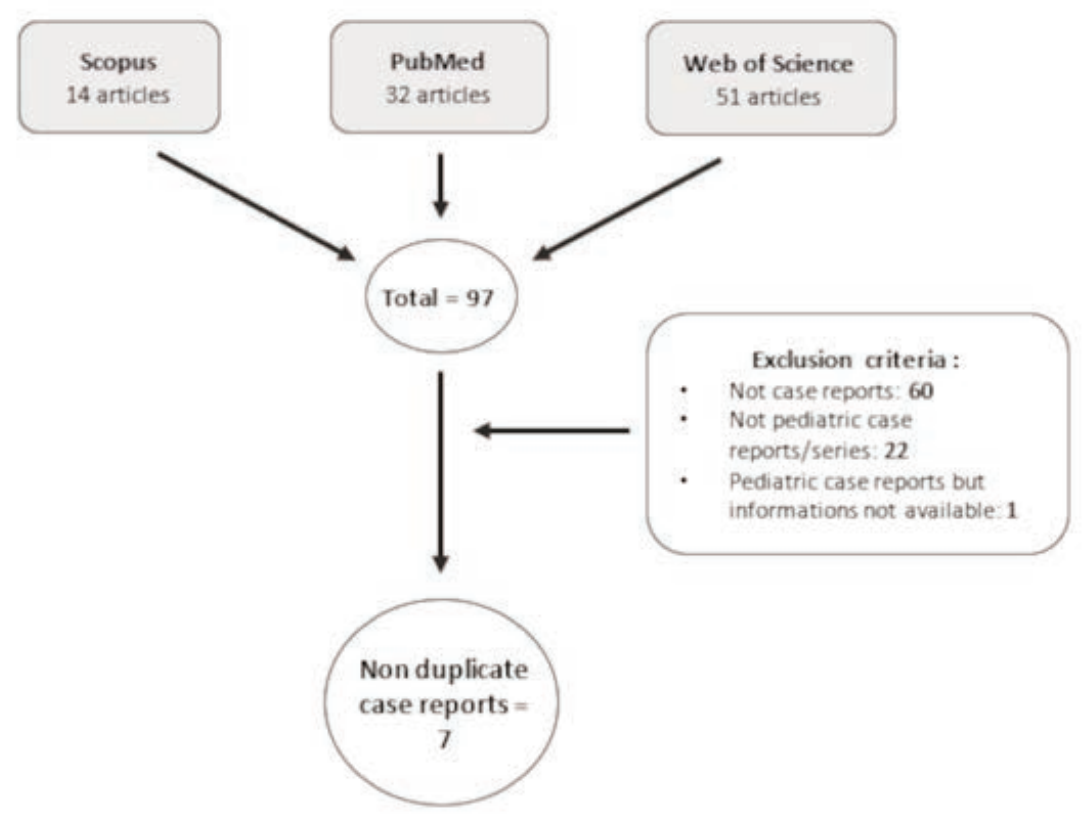

Figure 3. Literature review flowchart.

tion and/or cauterization of the lesion have been performed in the past, ${ }^{11}$ even though the risk of unaesthetic scars is quite higher. Conversely, laser treatment is extensively used in the adult for the treatment of isolated PWS and PG, and it was the treatment of choice in one child only. ${ }^{4}$ Arguably, laser treatment has been reported as a risk factor for PG development on PSW, and therefore its role is still debated. ${ }^{12}$

\section{Conclusions}

In conclusion, pyogenic granuloma arising on port-wine stains is a known entity, especially in the adult carrying risk factors. This association is rarer but probably underreported in children, and moreover it seems to be less linked to predisposing conditions. Although there is no agreement in the treatment of choice, we believe that complete excision of the vascular malfor- mation is crucial to prevent any recurrence and it should always be performed whenever possible. Nonetheless, radical treatment may be infeasible in a single attempt, especially for extended PWS. Thus, every case should be discussed with Dermatologists and/or a multidisciplinary group in order to define the best therapeutic plan.

1. Shruti S, Siraj F, Ramesh V, Ramesh V. Recurrent pyogenic granuloma over nevus flammeus. Indian J Dermatol Venereol Leprol 2019;85:236.

2. da Silva AD, Silva CA, de Camargo Moraes P, et al. Recurrent oral pyogenic granuloma in port-wine stain. $\mathrm{J}$ Craniofac Surg 2011;22:2356-8.

3. Swerlick RA, Cooper PH. Pyogenic granuloma (lobular capillary hemangioma) within port-wine stains. J Am

\section{References}

Acad Dermatol 1983;8:627-30.

4. Yang C, Liu S. Treatment of giant pyogenic granuloma with the $\mathrm{Nd} / \mathrm{YAG}$ holmium laser: a case report. J Cosmet Laser Ther 2013;15:225-7.

5. Weibel L. Vascular anomalies in chil dren. Vasa 2011;40:439-47.

6. Garzon MC, Enjolras O, Frieden IJ. Vascular tumors and vascular malformations: Evidence for an association. J Am Acad Dermatol 2000;42:275-9.

7. Holloway KB, Ramos-Caro FA, Brownlee RE Jr, Flowers FP. Giant proliferative hemangiomas arising in a port-wine stain. J Am Acad Dermatol 1994;31:675-6.

8. Sheenan DJ, Lesher JL. Pyogenic granuloma arising within a port-wine stain. Cutis 2004;73:175-80.

9. Lee JB, Kim M, Lee SC, Won YH. Granuloma pyogenicum arising in an arteriovenous haemangioma associated with a port-wine stain. Br J Dermatol 2000;143:669-71.

10. Chen D, Hu XJ, Lin XX, et al. Nodules arising within port-wine stains: a clinicopathologic study of 31 cases. Am J Dermatopathol 2011;33:144-51.

11. Abd-el-Raheem TA, Hohenleutner U, Landthaler M. Granuloma pyogenicum as a complication of flashlamp-pumped pulsed dye laser. Dermatology 1994;189:283-5.

12. Hagiwara K, Uezato $H$, Nonaka S. Phacomatosis pigmentovascularis type $\mathrm{IIb}$ associated with Sturge-Weber syndrome and pyogenic granuloma. J Dermatol 1998;25:721-9.

13. Warner J, Jones EW. Pyogenic granuloma recurring with multiple satellites. A report of 11 cases. Br J Dermatol 1968;80:218-27.

14. Liu S, Yang $\mathrm{C}, \mathrm{Xu} \mathrm{S}$, et al. Pyogenic granuloma arising as a complication of $595 \mathrm{~nm}$ tunable pulsed dye laser treatment of port-wine stains: report of four cases. Dermatol Surg 2010;36:1341-3. 